\title{
Research forecasting model to solve the problem of marketing needs of enterprises
}

\author{
MO Jing-lan, WU Yang-yang, WANG Yao, HUANG Yu-ting \\ Department of Mathematics and Physics, Liuzhou Institute of Technology, Liuzhou, 545616, Guangxi, China
}

\begin{abstract}
This essay focuses on the temperature variation law of the center of the target board soldering area at a certain position when the reflow oven starts working as the core discussion. Using the principle of heat conduction, Fourier's law to solve the problem of temperature values and temperature change laws in the center of the soldering area of the target circuit board at different locations under set conditions. As in the actual process, the temperature change in the center of the soldering area is limited to certain conditions, we can use the target planning, prediction methods, to build a model to solve the maximum problem. At the same time we need to consider the quality of the target circuit board, the use of neural networks, genetic algorithms to establish optimization models for target optimization, and ultimately the optimal furnace temperature curve.
\end{abstract}

\section{Introduction}

As technology continues to progress and develop, the variety and quantity of electronic products is increasing, and electronic product manufacturers have extremely high requirements for the reliability of their production products. The quality and reliability of the Print Circuit Board Assembly (PCBA) of electronic products is mainly reflected in the Surface Mounted Technology (SMT) reflow soldering link ${ }^{[1-2]}$. Quality improvement in reflow soldering is primarily a matter of improving the reflow output oven temperature profile. Therefore, it is very important to study the reflow soldering temperature profile optimization and get a temperature profile that is applicable to the requirements of the equipment.

Feng Zhigang et al. ${ }^{[3]}$ conducted a comprehensive study on the influence of technological parameters of reflow welding furnace on the reflow temperature curve and its key indexes by using orthogonal experimental method, and pointed out that the conveyor belt speed and furnace temperature in welding area had the most obvious influence. Li Yan et al. ${ }^{[4]}$ optimized the reflow oven temperature parameters by calculating the heating factor. Zhu Guibing et al. ${ }^{[5]}$ combined thermal efficiency and PCBA assembly density to determine the cooling rate of the temperature profile, and the deficiencies of the temperature profile settings were inferred by inverse welding defects combined with the concept of thermal efficiency to seek an optimization approach for the oven temperature profile. Gong Yubing ${ }^{[6]}$ made heating factor, maximum temperature and other factors as constraints, the use of genetic algorithms to find the optimal maximum thermal stress is reduced. And Wan Xue-bin ${ }^{[7]}$ did a fitting study on reflow welding temperature curve. Reflow soldering furnace temperature curve for numerical analysis of optimization research is relatively smal[ ${ }^{[8-11]}$, this essay focuses on reflow soldering furnace temperature change analysis, using the principle of heat conduction, Fourier's law to solve the problem of temperature values and temperature change patterns in the center of the target circuit board soldering area at different locations under set conditions. In the center of the soldering area temperature change is limited to certain conditions, the use of target planning, forecasting methods to find the maximum value of the oven temperature change. Also consider the quality of the target circuit board, the use of neural networks, genetic algorithms to find the optimal furnace temperature curve.

\section{Reflow oven process flow}

In the reflow oven, both sides of the target circuit board are placed on a conveyor belt and processed into the oven at a uniform speed. The specific structure design, the length of each workpiece and other data as shown in Figure 1. After the reflow oven starts working, its internal air temperature will be quickly stabilized in a short period of time, and then the target circuit board can be processed. No special temperature control is done for the areas and gaps where the initial temperature is not set. The gap temperature changes due to the influence of adjacent temperature zones, and the temperature around the boundary of each temperature zone may also change. Note: The shop temperature should be maintained at $25^{\circ} \mathrm{C}$.

\footnotetext{
*E-mail: jinglan022@163.com
} 


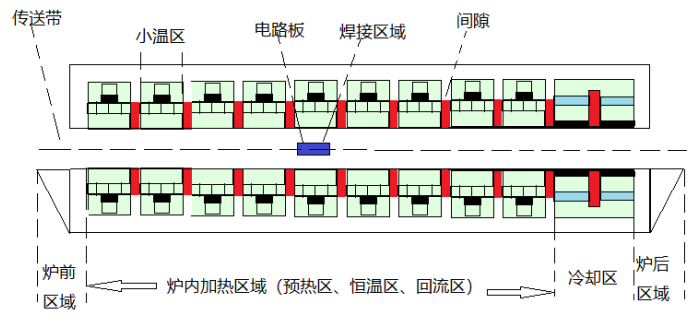

Fig. 1 Section diagram of furnace

Using a temperature sensor to measure the center temperature of the target circuit board soldering area at a specific location, the resulting curve of the temperature change pattern is called the center temperature curve of the soldering area (i.e., the oven temperature curve). To an experiment, for example, the initial setting of the temperature of each small temperature zone: $1-5\left(175^{\circ} \mathrm{C}\right)$, $6\left(195{ }^{\circ} \mathrm{C}\right), 7\left(235^{\circ} \mathrm{C}\right), 8-9\left(255^{\circ} \mathrm{C}\right)$ and $10-11\left(25^{\circ} \mathrm{C}\right)$; target circuit board soldering area thickness of $0.0015 \mathrm{~cm}$; conveyor belt through the reflow speed of $70 \mathrm{~cm} / \mathrm{min}$. Whenever the center of the target circuit board soldering area reaches $30^{\circ} \mathrm{C}$, the temperature sensor starts to work, and the timing work starts when the target circuit board goes into the reflow oven.

Assuming the temperature as the basis, each small temperature zone can be adjusted for the set temperature in the range of $10{ }^{\circ} \mathrm{C}$ up and down. Which requires the adjustment process small temperature zone (1-5), (8-9), (10-11) between the temperature adjustment range to maintain consistent. Target circuit board through the reflow oven, the conveyor speed can be adjusted within $65-100 \mathrm{~cm} / \mathrm{min}$. When producing reflow oven circuit boards at the same time, the oven temperature profile will meet the manufacturing limits.

\section{Reflow oven numerical simulation and results}

\subsection{Temperature variation analysis}

According to the actual data, the general temperature profile of the reflow soldering working process is shown in Figure 3. Through the analysis of the temperature curve in Figure 3, it can be seen that the temperature of the center of the target board soldering area is constantly changing, the actual working temperature is not consistent with the initial set temperature and there is a certain deviation. Influenced by the adjacent temperature zone, the gap temperature changes. From the actual data, it is not difficult to find the target board has reached 30 degrees Celsius before entering the temperature zone, indicating that the temperature around the boundary of each temperature zone changes.

\subsection{Thermal conductivity analysis}

The gap temperature changes due to the influence of the adjacent temperature zone. Considering that the gap length is small, there is a certain working time and the temperature at both ends is high, we can assume that the gap temperature and the temperature of the adjacent temperature zone is approximate. When the target board into the reflow oven processing, assuming that the target board moves to a certain point, at this time seen as stationary state does not occur relative displacement, and the target board temperature at both ends of the inconsistent existence of temperature differences. Small temperature zone around the existence of thermal field curve, due to the reflow furnace work with thermal convection phenomenon, the target board side temperature is lower than the small temperature zone working surface, and there is a certain amount of heat flux $\Phi$ through the target board, at this time it can be seen as a onedimensional thermal conductivity, the specific physical plane diagram shown in Figure 2.

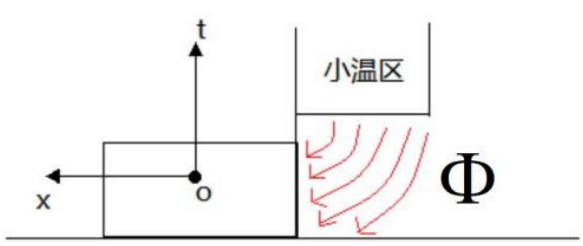

Fig. 2 Physical plan of heat conduction

It can be seen from the figure that the direction of heat transfer is opposite to the direction of temperature increase, which satisfies the second law of thermodynamics. Using the relevant experimental theorem, the temperature dynamic change model can be established, and the specific establishment process is shown in Figure 3.

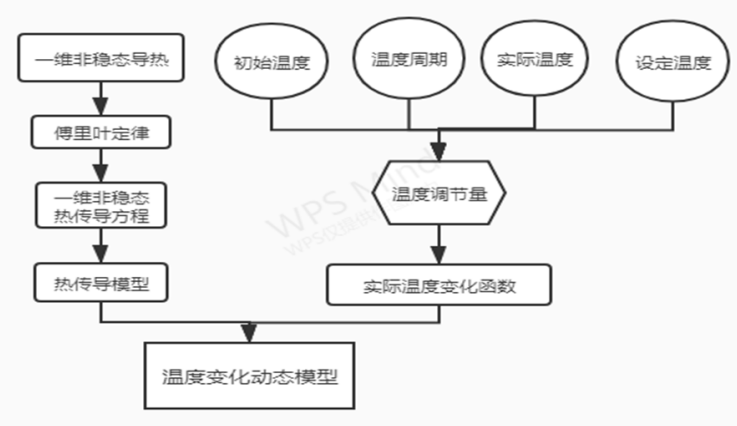

Fig. 3 Temperature dynamic change model

\subsection{Reflow oven model}

\subsubsection{Heat conduction model based on Fourier's law}

Fourier's law is the most applicable basic law in thermal science in heat transfer experiments. When the reflow oven starts working, the internal air temperature will quickly reach stability in a short period of time, assuming that the target board enters the reflow oven processing with a stable temperature change in the center of the soldering area. With a period of $0.5 \mathrm{~s}$ as the unit time, the model is constructed using Fourier's law.

One-dimensional unsteady thermal conductivity:

(1) Initial conditions: $\left.u\left(T_{c}, t\right)\right|_{t=0}=f\left(T_{c}\right)$, 
(2) Boundary conditions. $-\left.k \frac{\partial u}{\partial n}\right|_{p}=\left.Q\left(u-u_{1}\right)\right|_{p}$, where thermal conductivity is $k$, heat transfer coefficient is $Q$, target board boundary is $p$, target board soldering area boundary temperature is $u$, thermal conductivity medium temperature is $u_{1}$.

(3) One-dimensional non-stationary heat conduction equation.

Heat flux: $\Phi=\lambda S \frac{T_{w_{(i)}}-T_{w_{(i-1)}}}{d}$,

Which is the thickness of the weld area d, surface area quantification $\mathrm{S}$, stable wall temperature on both sides $T_{w_{(i)}} 、 T_{w_{(i-1)}}$.

Heat flux density: $M=\lambda \frac{T_{w_{(i)}}-T_{w_{(i-1)}}}{d}$.

Assuming that the workpiece in the reflow oven is thermally inertia-free, the heat change curve of the workpiece is close to the heat distribution curve transmitted to the surrounding area, so the approximate range of thermal conductivity is determined $\lambda_{i}(30 \sim 175)$.

Using the weighted average method, the range of thermal conductivity is determined. It is assumed that the variation with $\mathrm{x}$ between nodes $\mathrm{A}$ and $\mathrm{C}$ is approximately linearly distributed, and the relationship between the specific interface and nodes is shown in Figure 4.

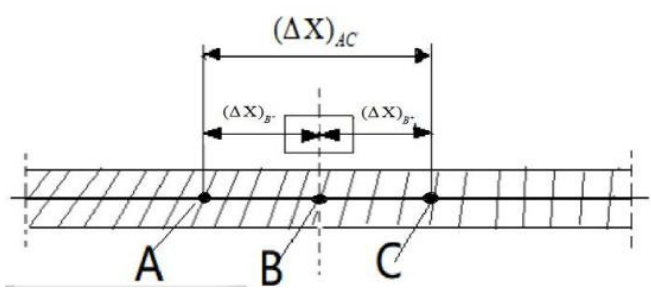

Fig. 4 Interface and node relationship diagram

$$
\begin{gathered}
\text { From Figure 4, we can get: } \\
\lambda_{B}=\left[\frac{(\Delta x)_{B^{+}}}{(\Delta x)_{B^{-}}}\right] \lambda_{A}+\left[\frac{(\Delta x)_{B^{-}}}{(\Delta x)_{B}}\right] \lambda_{C} .
\end{gathered}
$$

According to the above, at this time take $\lambda_{C} \approx 175^{\circ} \mathrm{C}, \lambda_{A} \approx 30^{\circ} \mathrm{C}$, bring it into the above equation can be obtained: $\lambda_{B}=\lambda_{A}\left[\frac{(\Delta x)_{B^{*}}}{(\Delta x)_{B}}\right]$, the heat conduction

equation can be obtained: $\quad \rho c \frac{\partial T}{\partial x}=\frac{1}{S} \frac{\partial}{\partial x}\left[\lambda S \frac{\partial T}{\partial x}\right]+T_{0}$, because the workpiece in the temperature zone is no thermal inertia workpiece, it is obtained: $R(t)=\frac{1}{S} \frac{\partial}{\partial x}\left[\lambda S \frac{\partial T}{\partial x}\right]+T_{0}$,

Heat transfer model: $\left\{\begin{array}{l}\left.u\left(T_{c}, t\right)\right|_{t=0}=f\left(T_{c}\right) \\ -\left.k \frac{\partial u}{\partial n}\right|_{p}=\left.Q\left(u-u_{1}\right)\right|_{p} \\ \Phi=\lambda S \frac{T_{w_{(i)}}-T_{w_{(i-1)}}}{d} \\ M=\lambda \frac{T_{w_{(i)}}-T_{w_{(i-1)}}}{d} \\ \lambda_{B}=\lambda_{A}\left[\frac{(\Delta x)_{B^{+}}}{(\Delta x)_{B}}\right] \\ \rho c \frac{\partial T}{\partial x}=\frac{1}{S} \frac{\partial}{\partial x}\left[\lambda S \frac{\partial T}{\partial x}\right]+T_{0} \\ R(t)=\frac{1}{S} \frac{\partial}{\partial x}\left[\lambda S \frac{\partial T}{\partial x}\right]+T_{0}\end{array}\right.$

\subsubsection{Dynamic model of temperature change}

Set the temperature period to $T=0.5 \mathrm{~s}$, actual temperature $R(t)$, set temperature $D(t)$ 、 temperature regulator $E(t)$, the initial temperature $T_{0}$, Start to build dynamic model with actual data.

temperature regulator $E(t)$ :

$$
E(t)=\int[D(t)-R(t)] d t+T_{i} \frac{d[D(t)-R(t)]}{d t},
$$

the nth input regulation amount is, then:

$$
E(n)=\sum_{t=0}^{n}\left[D_{k}(t)-R_{k}(t)\right]+\frac{\left(D_{n}-R_{n}\right)-\left(D_{n-1}-R_{n-1}\right)}{T},(k \text { take } 1,2,3 \ldots),
$$

The actual temperature can be obtained $R\left(t_{i}\right)$ :

$$
R\left(t_{i}\right)=T_{0}+E(n) \cdot T_{i}
$$

combined with the above equation to obtain:

$$
R\left(t_{i}\right)=T_{0}+T_{i} \sum_{t=0}^{n}\left[D_{k}(t)-R_{k}(t)\right]+T_{i} \frac{\left(D_{n}-R_{n}\right)-\left(D_{n-1}-R_{n-1}\right)}{T} .
$$

Using MATLAB software to solve the model, the temperature at the midpoint of the small temperature zone and the end of the small temperature zone at the center of the target circuit board soldering area can be derived, as shown in Table 1. Also solve for the temperature at the center of the soldering area of the target board for the time period.

Table 1 Welding center temperature results

\begin{tabular}{|c|c|c|c|c|}
\hline $\begin{array}{c}\text { Temperature } \\
\text { measuring } \\
\text { position }\end{array}$ & $\begin{array}{c}\text { Small temperature } \\
\text { region 3 midpoint }\end{array}$ & $\begin{array}{c}\text { Small temperature } \\
\text { region6 midpoint }\end{array}$ & $\begin{array}{c}\text { Small temperature } \\
\text { region 7 midpoint }\end{array}$ & $\begin{array}{c}\text { End of small } \\
\text { temperature zone 8 }\end{array}$ \\
\hline time $/ \mathrm{s}$ & 111.2 & 217.8 & 253.5 & 304.2 \\
\hline temperature $/{ }^{\circ} \mathrm{C}$ & 131.8 & 165.9 & 176.1 & 211.5 \\
\hline
\end{tabular}

To plot the furnace temperature curve, it is necessary to use software to program the data of the model, and the best way to program the plotting is to use the program flowchart, and organize the logical sequence of the plotting clearly. Therefore, based on the dynamic model of temperature change, the furnace temperature curve plotting program design, the specific procedures are as follows:

Input: set temperature $S(t)$, timing time value $m$. 
Step 1: Read the initial temperature $T_{0}$;

Step 2: then $n=1$;

Step 3:

$E(n)=S(n)-T(n)+[S(n)-R(n)]-[S(n-1)-R(n-1)] ;$

Step 4: $R(t)=T_{0}+E_{n} \cdot T_{n}$;

Step 5: then $n=n+1$;

Step 6: When $n \leq m$, return to step 3, otherwise execute the next step;

Step 7: Output $R(t)$.

The actual required furnace temperature curve can be obtained using MATLAB software, as shown in Figure 5.

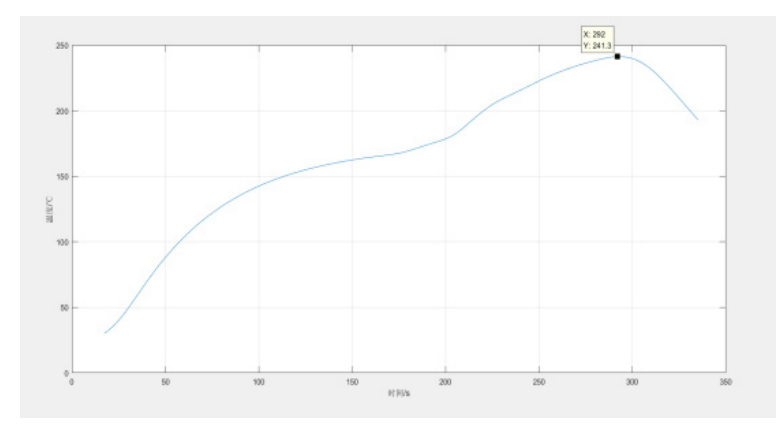

Fig. 5 Furnace temperature curve

Combined with the above results, the temperature results from the center of the target circuit board soldering area thought the time period to extract the target results for examination, the extraction boundaries results are shown in Table 2.

Table 2 Extraction target results

\begin{tabular}{|c|c|}
\hline Extract the name & results \\
\hline Time between $150^{\circ} \mathrm{C} \sim 190^{\circ} \mathrm{C}$ as the temperature rises $/(S)$ & $115.5-212$ \\
\hline Time when the temperature is greater than $217^{\circ} \mathrm{C} /(S)$ & $242.5-325.3$ \\
\hline Peak temperature $/\left({ }^{\circ} \mathrm{C}\right)$ & 241.3 \\
\hline
\end{tabular}

Nonlinear programming models are often used to solve optimization problems with one or several nonlinear functions in the objective function or constraints, which can better reflect the optimal solution. In the production process of IC boards, from entering the furnace to leaving the reflow oven, the variation between each large temperature is nonlinear, and there is also interaction between the temperature zones. The speed of the conveyor belt is influenced by the oven temperature profile while also needing to meet the process boundary limits.

Due to the variation of the amount of temperature change over time, it can be divided into the following four time periods according to their trends; the first time period (0-115.5), the second time period (115.5-197.5), the third time period (197.5-292.5), and the fourth time period (292.5-final) are treated separately.

Since the target circuit board welding area is uniform motion, it is known that time is proportional to displacement, so the relationship between displacement and the amount of temperature change can be obtained, as shown in Figure 6 below.

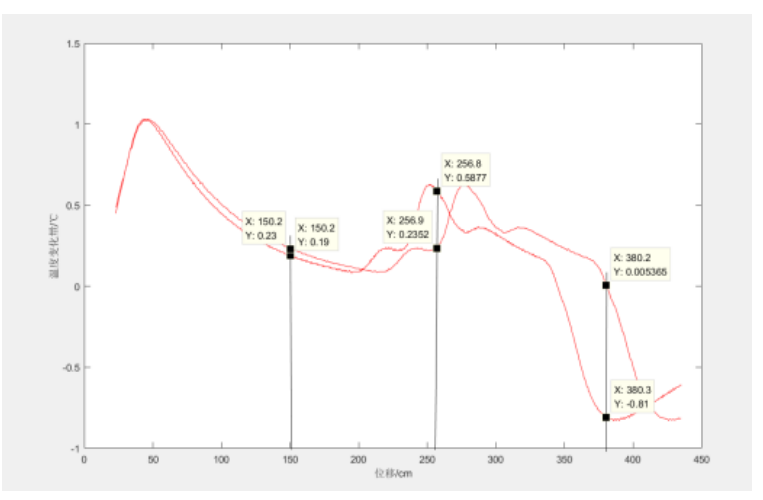

Fig. 6 Plot between displacement and the amount of temperature change

The amount of temperature change versus time for the first time period was deduced by fitting the data to MATLAB software and the results are shown in Figure 7 below.

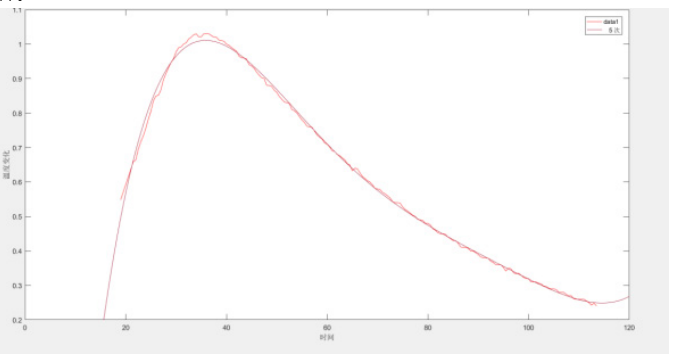

Fig.7 Plot of the amount of temperature change versus time for the time period

It can be obtained that

$k_{1}=0.029591 t_{1}^{5}-0.0746174 t_{1}^{4}+0.0095037 t_{1}^{3}+0.13583 t_{1}^{2}-0.35782 t_{1}+0.62234$

Similarly, it can be deduced that $k_{2}=0.0012705 t_{2}^{3}-0.60178 t_{2}^{2}+126.44 t_{2}-0.9941 .5$.
From the actual data to the process boundaries brought into the model can be obtained as follows: 


$$
\left\{\begin{array}{l}
L_{\text {炉长 }}=V\left(\mathrm{t}_{1}+\mathrm{t}_{2}+\mathrm{t}_{3}+\mathrm{t}_{4}\right), 60 \leq t_{3} \leq 120,40 \leq t_{4} \leq 90 \\
k_{1}=0.029591 t_{1}^{5}-0.0746174 t_{1}^{4}+0.0095037 t_{1}^{3}+0.13583 t_{1}^{2}-0.35782 t_{1}+0.62234,-3 \leq k_{2} \leq 0 \\
k_{2}=0.0012705 t_{2}^{3}-0.60178 t_{2}^{2}+126.44 t_{2}-0.9941 .5,-3 \leq k_{2} \leq 0
\end{array}\right.
$$

The result from MATLAB programming is that the maximum speed of the conveyor belt over the furnace at the specified temperature satisfying the process limit is: $V_{\max }=87.73 \mathrm{~cm} / \mathrm{min}$.

\section{Reflow oven temperature profile optimization}

\subsection{Optimization of furnace temperature profile based on neural network}

Neural networks are better able to obtain a non-linear mapping relationship between the input and output values of the furnace temperature curve. The target circuit board processing process, temperature variation process nonlinear, qualified circuit board soldering degree of good or bad need to maintain within a certain range of feature values. Neural network is able to generate functional relationships by learning and storing a large amount of input and output data to solve for a range of feature values under specific conditions. The known parameters can be divided into 3 layers, i.e., input layer, output layer, and intermediate layer (implicit layer), as shown in Figure 8.

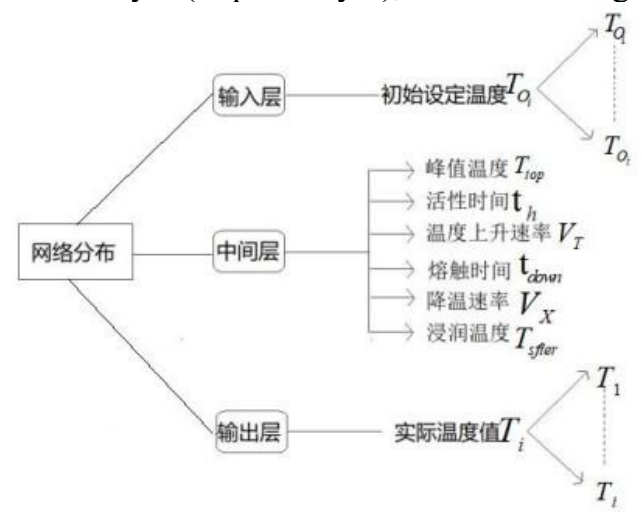

Fig. 8 Neural network distribution

The neural network learns and adapts to the original data in a certain direction. When the learning is completed, the unit activation values of the neurons will be passed from each implicit layer of the input layer to the output layer, and the neuron output and input in the output layer will establish a functional relationship autonomously, and the output and the actual output error will be minimized by adjusting the weights.

The range of input eigenvalues is used to construct the objective function equation. Using neural network technology, the eigenvalues of the hidden layer are solved, at which point the optimal furnace temperature profile can be plotted. Considering the high temperature on the thickness of the target board soldering area and the melting rate of the solder paste, the heating factor model is established to finally produce the desired optimization results.
Assume that the initial temperature characteristic value takes a range of $\left[d_{k}, r_{k}\right], d_{k}, r_{k}$ is the upper and lower temperature limit, then for the eigenvalues $C_{k}$ to construct the objective function, the error function $F\left(C_{k}\right)$ is:

$$
F\left(C_{k}\right)=\left\{\begin{array}{l}
A\left[\frac{C_{k}-d_{k}}{d_{k}}\right]^{2}, C_{k}<d_{k}, \\
0, d_{k} \leq C_{k} \leq r_{k} \\
A\left[\frac{C_{k}-r_{k}}{r_{k}}\right]^{2}, C_{k}>r_{k}
\end{array},\right.
$$

Where $A$ is the proportionality constant taken as 1 . The sum of the eigenvalues is used as the optimization objective function of the furnace temperature curve:

$$
\text { Miny }=\sum_{k=1}^{Y} F\left(C_{k}\right),(k=1,2 \ldots),
$$

At this point the activation function: $\varphi(w)=\frac{1}{1+e^{-T_{i} w}}, \varphi(w)=\frac{1-e^{-w}}{1+e^{-w}}$, the neural network structure, as shown in Figure 9.

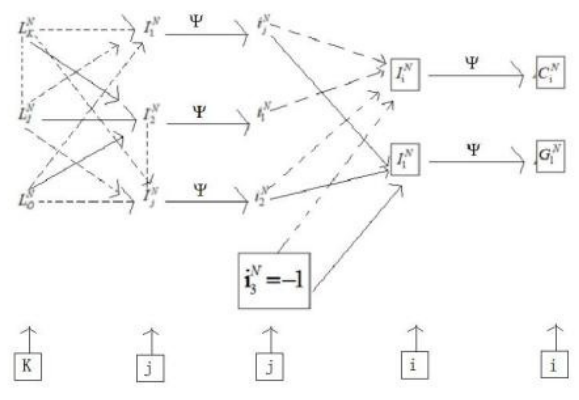

Fig. 9 Structure of neural network

According to the structure of the knotted network, the original data is input for training, and we get:

$$
C_{i}^{N}=\varphi\left[\sum_{j=0}^{6} G_{i j} \varphi\left(\sum_{k=0}^{10} \overline{G_{i j}} L_{k}^{N}\right)\right] \cdot
$$

The optimization model can be complemented by a model using the heating factor, considering the effect of the heating factor on the temperature profile. The heating factor $Q_{\beta}$ is $Q_{\beta}=\frac{\Delta t-\Delta T}{\pi} \int_{t_{1}}^{t_{2}}\left[T_{i}(t)-T_{t o p}\right] d t$.

Where $T_{t o p}$ is the peak temperature, the melting point temperature of the solder (Note: the peak temperature should not exceed the melting point temperature of the solder for too long). $\Delta t=t_{1}-t_{2}$ for the time beyond $T_{L}=217^{\circ} \mathrm{C}$, which corresponds to the area above the time region on the stay, as shown in Figure 10. 


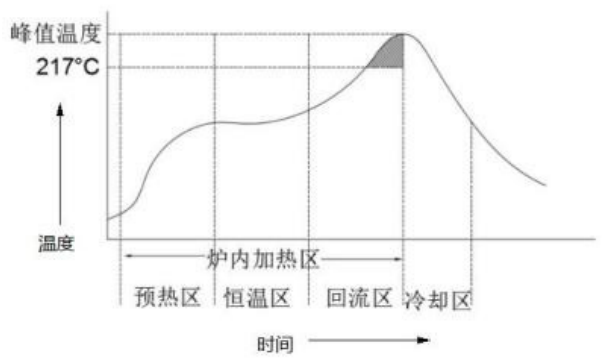

Fig.10 Heating factor definition diagram

Approximate the shaded area part as a single half-wave, as shown in Figure 11:

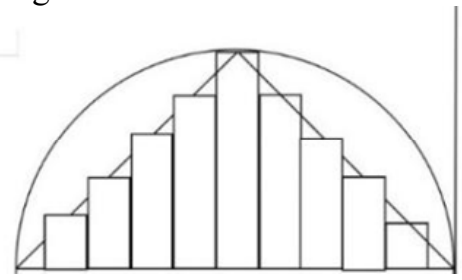

Fig.11 Approximate graph

Based on the graph, this can be simplified to $Q_{\beta}=\frac{\Delta t-\Delta T}{\pi} \int_{0}^{\pi} \sin t d t \quad, \quad$ where $\quad \Delta t=t_{1}-t_{2}$, $\Delta T=T_{\text {top }}-T_{L}$.

From the above it can be seen that at this time the heating factor temperature $T_{L}$ of the solder paste approximately in the substitution model can be obtained area 467 , conveyor belt over the solder furnace speed of $83 \mathrm{~cm} / \mathrm{min}$, the temperature of each temperature zone: small temperature zone $1-5$ temperature of $186 /{ }^{\circ} \mathrm{C}$, small temperature zone 6 temperature of $205 /{ }^{\circ} \mathrm{C}$, small temperature zone 7 temperature of $233 /{ }^{\circ} \mathrm{C}$, small temperature zone 8 temperature of $256 /{ }^{\circ} \mathrm{C}$.

\subsection{Optimization of furnace temperature profile based on genetic algorithm}

Although the neural network achieves good results in the nonlinear shadowing relationship between the input and output values of the temperature profile, the raw data of the hidden unit in the implicit layer has a certain error, so that the furnace temperature profile made cannot be optimized. Therefore, this section introduces a genetic algorithm to optimize the objective of the raw data in the hidden layer so as to achieve the optimal state.

Real number coding of the temperature profile of the indicator parameters. A solution after encoding is called a chromosome. There are four gene values on the chromosome, which are the temperatures of the four temperature zones in turn. The target board enters the reflow oven speed and soldering area thickness, where the set temperature and speed are controllable parameters and the heating factor cannot be controlled.

The fraction with the smallest variance parameter is selected as the initial population, and the product variance parameter $E_{\eta}$ is

$$
E_{\eta}=K_{g}\left(Q_{4}^{n}-Q_{4}^{\wedge}\right)+\sum_{k=1}^{Y} F\left(C_{k}\right) .
$$

Where $Q_{4}^{n}$ is the heating factor of the nth training sample, $\hat{Q_{4}}$ is the new heating factor, and $K_{g}$ is the scale factor can take the value of 1 .

The fitness function is a functional relationship that measures the fitness of individuals in a population. It can be used to measure the degree of excellence of each individual in the population, and after genetic processing the fitness function $f\left(Q_{i}\right)$ can be obtained as $f\left(Q_{i}\right)=\frac{1}{1+e}$.

Genetic processing is performed by algorithmic crossover and non-uniform variation, and the core element in the genetic algorithm is the genetic processing factor. Therefore, the selection of chromosomes is crucial, and algorithmic crossover is more realistic compared to other crossover methods, and can largely reduce the loop breaking of chromosomes. Assuming that $y_{1} 、 y_{2}$ are a certain gene locus of two randomly selected individuals in the parent population, the corresponding gene values within the offspring individuals are

$$
\left\{\begin{array}{l}
y_{1}^{\prime}=b y_{1}+(1-b) y_{2} \\
y_{2}^{\prime}=b y_{2}+(1-b) y_{1}
\end{array} .\right.
$$

Where $b$ is the constant between $[0 \sim 1]$.

The non-uniform variation operator is more precisely fine-tuned locally as the transmission changes. Assuming that the gene at variation point temperature $Q_{i}$ takes a range of values $\left[Q_{i}^{\min }, Q_{i}^{\max }\right]$, the new gene takes a value $Q_{i}^{\prime}$ determined by the following equation:

$$
Q_{i}^{\prime}=\left\{\begin{array}{l}
Q_{i}+\Delta\left(\tau, Q_{i}^{\max }-Q_{i}\right), \text { random }(0,1)<0.5 \\
Q_{i}-\Delta\left(\tau, Q_{i}-Q_{i}^{\mathrm{min}}\right), \operatorname{random}(0,1) \geq 0.5
\end{array} .\right.
$$

Denote in $\Delta\left(\tau, Q_{i}^{\max }-Q_{i}\right) 、 \Delta\left(\tau, Q_{i}-Q_{i}^{\min }\right)$ random values $[0, Q]$ that conform to a non-uniform distribution in the range $\Delta\left(\tau, Q_{i}^{\max }-Q_{i}\right) 、 \Delta\left(\tau, Q_{i}-Q_{i}^{\min }\right)$, and can be expressed as $\Delta\left(t, Q_{z}\right)$ and whose limit tends to 0 ,

$$
\Delta\left(\tau, Q_{z}\right)=Q_{z}\left(1-b^{\left(1-\frac{1}{T}\right) \alpha}\right),
$$

Where $b$ is a uniformly distributed constant in the range $[0,1], \tau$ is the algebraic value that has evolved, $\alpha$ is the coefficient parameter, and the range of values of $\left[Q_{i}^{\min }, Q_{i}^{\max }\right]$ is consistent with the range of temperature eigenvalues in Problem 3.

The rate of evolution is advanced by selecting treatments according to the benefits of the degree of adaptation. Assume that the population size is such that when the fitness value of a chromosome is $f\left(Q_{i}\right)$, the probability of the $i$ th chromosome being selected is

$$
P_{\left(Q_{i}\right)}=\frac{f\left(Q_{i}\right)}{\sum_{i=1}^{N} f\left(Q_{i}\right)} .
$$

The above model is solved using MATLAB software, 
and the solved descendants are substituted into the neural network training as the original data of the hidden layer to optimize the neural network-based furnace temperature optimization model, and the optimal model is derived. The optimal furnace temperature curve while super $217^{\circ} \mathrm{C}$ furnace temperature curve is highly symmetrical with the peak temperature dividing the centerline. The model is realistic and effective, and the optimization effect is good.

\section{Conclusion}

The use of nonlinear programming model for interactive fitting of the results by extrapolation can minimize the error brought about by linearization, the results are in line with the true range, but the computational effort is too large for optimization models; the model using neural network technology, with highly accurate classification techniques and powerful parallel distribution capabilities, can fully approximate the nonlinear relationship. Using the heating factor and genetic factor model to adjust the reflow oven set temperature in each temperature zone and the target board through the reflow oven speed, the model has a reference worth promoting; genetic algorithm based on neural networks to build optimization models, plot the optimal oven temperature curve. Optimization prediction results are obtained by suitable genetic factors.

\section{Biography}

Mo Jinglan was born in 1984. She is a associate professor. Her research interests include Rough Sets and Data Mining.

\section{Acknowledgments}

The author would like to thank the valuable suggestions from the anonymous reviewers and Professor. This research was supported by the basic ability improvement project of middle and young teachers in colleges and universities of Guangxi (No.2018KY0869 and 2019KY1098), the Natural Science Foundation of Guangxi Province of China (No.2019GXNSFAA245031).

\section{References:}

1. Pan Kailin, Zhou Dejian, Qin Kuangyu. Research status of SMT reflow welding Process Prediction and Simulation Technology [J]. Electronic Process Technology, 2000(5): 185-187.

2. Mao Xinlong, Han Guoming, Huang Bingyuan, et al. Modeling and Simulation of reflow welding process in SMT [J]. Welding technology, 2004,33 (5): 17-20.

3. Feng Zhigang, Yu Tingwen, Zhu Yunhe. Influence of reflow welding parameters on temperature curve [J]. Electronic Technology, 2004, (6): 243-246.

4. Li Yan, Zhao Libo, Zhang Wei, Qiu Zhaoyi. Analysis and Optimization of reflow welding furnace temperature Setting $[\mathrm{J}]$. Ship electric technology, 2010, 30(07): 44-46.
5. Zhu Guibing, Chen Wensuo, Zhao Xiongming. Research on the optimization of reflow welding temperature curve to prevent defects [J]. Hot working process, 2011,40 (19): 133-135, 138.

6. Gong yubing. Research on optimization of temperature curve of reflow welding furnace [J]. Thermal processing technology,2013,15(42): 187$190,193$.

7. Wan Xuebin. Fitting study of reflow welding temperature curve $[\mathrm{J}]$. Henan Science and Technology, 2008,11(12):53-54.

8. Fei Zemin. Setting of temperature distribution curve of reflow furnace [J]. Electronic Technology,1997, (04): 22-25.

9. Lei Xiangxiao, Tang Chunxia, Xu Lijuan. Temperature control of hot air reflow welding based on RBF-PID [J]. Journal of Shaoyang university (natural science edition),2020,17(04):31-38.

10. Sun Liqiang. PCB Reflow welding Temperature curve Setting optimization [J]. Electronic Technology and Software Engineering, 2014, (07): 150-151.

11. Guo Yu, Sun Zhili, Yuan Zhe, Pan Ershun. Parameter setting of reflow welding based on Neural Network Genetic Algorithm [J]. Mechanical science and technology,2013,32(08):1211-1214,1220. 